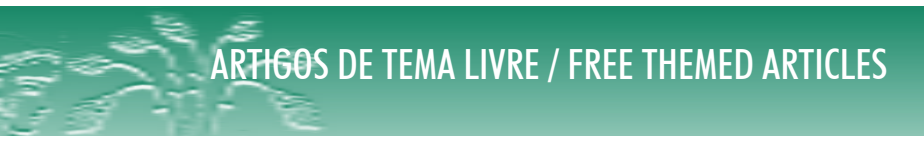

DOI: 10.12957/demetra.2018.30309

\title{
Comportamento alimentar e perfil nutricional de pacientes oncológicos em tratamento quimioterápico
}

\section{Feeding behavior and nutritional profile of cancer patients in drug therapy}

Pâmela Capelari'

Giovana Cristina Ceni'

' Universidade Federal de Santa Maria, Departamento de Alimentos e Nutrição. Palmeira das Missões, RS, Brasil.

Correspondência / Correspondence Pâmela Capelari

E-mail: pamelacapelari@hotmail.com

\section{Resumo}

Introdução: A alimentação tem grande importância na terapêutica oncológica, pois a morbimortalidade depende do perfil nutricional dos pacientes. Objetivo: Verificar o comportamento alimentar e o perfil nutricional de pacientes adultos em tratamento quimioterápico. Metodologia: Estudo transversal, de caráter quantitativo, desenvolvido em um Centro de Alta Complexidade em Oncologia no município de Ijuí-RS. Foram avaliados os pacientes oncológicos adultos, de ambos os sexos, no período de agosto a setembro de 2015 . As variáveis analisadas foram: características sociodemográficas e clínicas dos pacientes, comportamento alimentar, consumo dietético e avaliação antropométrica. Resultados: Participaram 100 pacientes, sendo a maioria do sexo feminino (56\%). O diagnóstico primário principal foi o de câncer de mama $(51,79 \%)$ entre as mulheres e de pulmão $(15,91 \%)$ entre os homens. Houve predomínio de aversões alimentares a carnes, alimentos gordurosos, doces e alimentos sólidos. A caracterização da alimentação devido ao tratamento foi estatisticamente significante $(\mathrm{p}=0,035)$. Indivíduos de ambos os sexos apresentaram consumo energético médio menor que $1.300 \mathrm{Kcal} / \mathrm{dia}$. O sobrepeso foi prevalente entre as mulheres $(44,64 \%)$ e a eutrofia, entre os homens $(45,45 \%)$. Conclusões: $\mathrm{O}$ tratamento quimioterápico alterou o comportamento alimentar dos pacientes, e o baixo consumo energético e proteico associado à perda de peso sugere possível risco nutricional.

Palavras-chave: Câncer. Comportamento alimentar. Estado nutricional. Quimioterapia. 


\section{Abstract}

Introduction: Feeding is extremely important in cancer therapy, and morbidity and mortality depend on the nutritional profile of patients. Objective: To verify the feeding behavior and nutritional profile of adult patients in drug therapy. Methodology: Crosssectional quantitative study, developed in a highly complex Oncology Center in the city of Ijuí-RS, Brazil. Adult cancer patients of both sexes were evaluated from August to September 2015. The analyzed variables were socio-demographic and clinical characteristics of patients, feeding behavior, dietary intake and anthropometric assessment. Results: One hundred patients participated, mostly women (56\%). The main primary diagnosis was breast cancer (51.79\%) among women and lung (15.91\%) among men. There was predominance of food aversions to meat, fatty foods, sweets and solid foods. The characterization of food due to treatment was statistically significant $(\mathrm{p}=0.035)$. Individuals of both sexes showed average energy consumption less than 1,300 Kcal/day. Overweight was prevalent among women (44.64\%) and normal weight among men (45.45\%). Conclusions: Drug therapy altered the feeding behavior of patients; low energy and protein intake associated with weight loss suggest possible nutritional risk.

Keywords: Cancer. Feeding behavior. Nutritional status. Drug therapy.

\section{Introdução}

O câncer apresenta origem multicausal crônica, sendo caracterizado pela propagação e desenvolvimento de modo descontrolado de células com o material genético alterado, o que propicia a formação de tumores neoplásicos em órgãos ou tecidos do organismo. ${ }^{1}$ Segundo a Organização Mundial da Saúde (OMS), a estimativa é que nos próximos 20 anos o número de novos casos de câncer aumente em aproximadamente $70 \%$, devido principalmente ao consumo de tabaco e de bebidas alcoólicas, má qualidade alimentar e inatividade física. ${ }^{2}$

A alimentação tem grande importância na terapêutica oncológica, devido a seus aspectos simbólicos, subjetivos e nutricionais; sendo assim, a análise do consumo alimentar de indivíduos com câncer deve ser uma prática rotineira. ${ }^{3}$ Estudos apontam que a qualidade de vida dos pacientes, posterior ao diagnóstico de câncer, sofre interferência das modificações que surgem nos padrões de ingestão decorrentes de aspectos nutricionais e dietéticos, como mudanças no paladar ou olfato, náuseas e mucosites orais, que provocam a redução da ingestão e podem repercutir no prognóstico clínico da doença. ${ }^{4,5}$ 
De acordo com Silva et al., ${ }^{6}$ efeitos adversos no trato gastrointestinal e anorexia são potencializados pela utilização de quimioterápicos. A quimioterapia antineoplásica tem por finalidade destruir os tumores malignos, por indução à apoptose ou inibição das atribuições básicas da célula; entretanto, por ser um tratamento sistêmico, não específico, pode atingir, além das células neoplásicas, as células normais. ${ }^{6,7}$

Deste modo, os pacientes podem apresentar alterações no comportamento alimentar, ${ }^{4,5}$ sendo um desafio complexo a manutenção dos hábitos alimentares cotidianos. ${ }^{5}$ A morbimortalidade depende diretamente do comprometimento do perfil nutricional dos pacientes, sendo que, quando o indivíduo se encontra desnutrido, há maior chance de infecção e redução dos resultados almejados com o tratamento. No entanto, ainda são escassos os estudos que analisam o estado nutricional de indivíduos com neoplasias associado ao consumo alimentar. ${ }^{3,8}$

Considerando que a oncologia é um problema atual e emergente da saúde pública, é de suma importância identificar a amplitude do tratamento quimioterápico nas condições de saúde dos pacientes, possibilitando evidenciar terapêuticas nutricionais que minimizem os impactos negativos e proporcionem melhora da qualidade de vida. ${ }^{9,10}$ Nessa perspectiva, o presente estudo objetivou verificar o comportamento alimentar e o perfil nutricional de pacientes adultos em tratamento quimioterápico em um Centro de Alta Complexidade em Oncologia localizado no noroeste do estado do Rio Grande do Sul.

\section{Metodologia}

Trata-se de estudo transversal, de caráter quantitativo, desenvolvido em um Centro de Alta Complexidade em Oncologia (CACON), localizado no município de Ijuí, noroeste do estado do Rio Grande do Sul, que presta assistência à população de 120 municípios da região.

A população de estudo foi composta por todos os pacientes oncológicos adultos, de ambos os sexos, em tratamento quimioterápico no período de agosto a setembro de 2015 , que concordaram em participar do estudo por meio da assinatura do Termo de Consentimento Livre e Esclarecido. Foram excluídos da pesquisa os indivíduos edemaciados e aqueles que estavam em tratamento para outras patologias, considerando que estes aspectos poderiam alterar os resultados do estudo.

A população de estudo foi abordada durante o processo de aplicação da quimioterapia e caracterizada através do prontuário e da aplicação de um questionário semiestruturado para coleta das seguintes informações: sexo, idade, estado civil, escolaridade, profissão, diagnóstico primário, metástase sistêmica, tratamentos propostos, peso usual e mudança de peso nos últimos seis meses.

Para a avaliação do perfil nutricional, foram utilizados métodos antropométricos e dietéticos. A avaliação antropométrica contemplou a aferição da circunferência da cintura, do peso corporal 
e da estatura dos indivíduos. Com os dados de peso e estatura, calculou-se o índice de massa corporal (IMC), que consiste no peso em quiilogramas, dividido pelo quadrado da altura em metros, empregando-se a classificação proposta pela OMS. ${ }^{11}$

Para a obtenção do peso corporal, foi utilizada uma balança digital da marca Plenna ${ }^{\circledR}$, com capacidade de $150 \mathrm{~kg}$, conforme as técnicas descritas por Nacif e Viebig. ${ }^{12}$ A estatura foi verificada de acordo com os procedimentos padrões descritos por Duarte, ${ }^{13}$ utilizando-se um estadiômetro portátil da marca Sanny ${ }^{\oplus}$, com capacidade de medição de 115 a $210 \mathrm{~cm}$.

A aferição da circunferência da cintura foi efetuada através da utilização de uma fita métrica inelástica e inextensível, da marca Cescorf ${ }^{\oplus}$, com precisão de um milímetro e dois metros de comprimento. A medida foi verificada no ponto médio entre a borda inferior da última costela e a crista ilíaca, ao final da expiração, ${ }^{13} \mathrm{e}$ classificada segundo a OMS,,${ }^{14} \mathrm{em}$ relação ao risco cardiovascular.

Como método de avaliação dietética, foi aplicado um recordatório alimentar de 24 horas, através do qual os indivíduos definiram e quantificaram em medidas caseiras todos os alimentos e bebidas ingeridos nas últimas 24 horas, ou mais comumente, no dia anterior, de acordo com o estabelecido por Fisberg et al..$^{15}$ Os valores nutricionais foram calculados utilizando-se a planilha de cálculos CalcNut ${ }^{\circledast}$, onde foram analisadas calorias totais e macronutrientes (carboidratos, proteínas e lipídeos).

A avaliação do comportamento alimentar foi realizada através de um questionário adaptado da avaliação subjetiva global pelo próprio paciente (ASG-PPP), desenvolvido com base em Silva et al., ${ }^{6}$ e no Inquérito Brasileiro de Nutrição Oncológica, ${ }^{10}$ contendo perguntas aos participantes sobre como e com quem se alimentavam, onde e quantas refeições realizavam, sobre aversões alimentares desenvolvidas durante o tratamento quimioterápico (existência ou não de algum alimento que o sujeito deixou de comer após iniciar a quimioterapia, por causar alguma forma de desconforto) e em relação a situações adversas que interferem na alimentação.

Para a análise estatística, utilizou-se o software EpiInfo ${ }^{\circledast}$ versão 7.0, considerando valores de $\mathrm{p}<0,05$ estatisticamente significantes. Para a avaliação de variáveis numéricas, foram empregados os testes paramétricos ANOVA e teste $t$ de Student e para variáveis categóricas, o teste QuiQuadrado $\left(\chi^{2}\right)$.

Esta pesquisa foi autorizada pelo Comitê de Estágios e Pesquisas da AHCI (Associação Hospital de Caridade de Ijuí), ao qual o Centro de Alta Complexidade em Oncologia (CACON) está vinculado, e posteriormente, submetida e aprovada pelo Comitê de Ética em Pesquisa da Universidade Federal de Santa Maria (UFSM), segundo a Resolução CNS 466/2012 do Conselho Nacional de Saúde, que regulamenta a pesquisa envolvendo seres humanos, sob o CAAE 46664815.7.0000.5346 e parecer 1.188.801. 


\section{Resultados}

Participaram da pesquisa 100 pacientes, dos quais 56\% eram do sexo feminino. Entre as mulheres, 48,21\% $(\mathrm{n}=27)$ eram aposentadas e 12,50\% $(\mathrm{n}=7)$, agricultoras, assim como 36,36\% $(n=16)$ dos homens relataram ser aposentados e 18,18\% $(n=8)$, agricultores. Na tabela 1, estão descritas outras características sociodemográficas da população estudada, sendo que a maioria dos pacientes eram casados (60,71\% das mulheres e 79,55\% dos homens) e apresentavam menos que oito anos de escolaridade (62,5\% das mulheres e 79,55\% dos homens).

As características clínicas estão demonstradas na tabela 2. Como diagnóstico primário, observou-se predomínio de casos de câncer de mama (51,79\%) entre as mulheres e de pulmão $(15,91 \%)$ entre os homens.

Tabela 1. Características sociodemográficas dos pacientes oncológicos. Ijuí-RS, Brasil, 2015.

\begin{tabular}{llccc}
\hline & & $\begin{array}{c}\text { Mulheres } \\
(\mathrm{n}=56)\end{array}$ & $\begin{array}{c}\text { Homens } \\
(\mathrm{n}=44)\end{array}$ & $\mathrm{p}$ \\
\hline Idade (anos)* & & $58,45 \pm 12,34$ & $59,89 \pm 13,30$ & $0,576^{* *}$ \\
\hline \multirow{2}{*}{ Estado civil } & Solteiro (a) & $1,79 \%(\mathrm{n}=1)$ & $13,64 \%(\mathrm{n}=6)$ & \\
& Casado (a) & $60,71 \%(\mathrm{n}=34)$ & $79,55 \%(\mathrm{n}=35)$ & $0,000^{* * *}$ \\
& Divorciado (a) & $10,71 \%(\mathrm{n}=6)$ & $4,55 \%(\mathrm{n}=2)$ & \\
& Viúvo (a) & $26,79 \%(\mathrm{n}=15)$ & $2,27 \%(\mathrm{n}=1)$ & \\
\hline & Analfabeto (a) & $7,14 \%(\mathrm{n}=4)$ & $6,82 \%(\mathrm{n}=3)$ & \\
& Fund. incompleto & $42,86 \%(\mathrm{n}=24)$ & $50,00 \%(\mathrm{n}=22)$ & \\
& Fundamental completo & $12,50 \%(\mathrm{n}=7)$ & $22,73 \%(\mathrm{n}=10)$ & \\
& Médio incompleto & $1,79 \%(\mathrm{n}=1)$ & $6,82 \%(\mathrm{n}=3)$ & \\
& Médio completo & $19,64 \%(\mathrm{n}=11)$ & $4,55 \%(\mathrm{n}=2)$ & $0,164 * * *$ \\
& Técnico & $1,79 \%(\mathrm{n}=1)$ & $2,27 \%(\mathrm{n}=1)$ & \\
& Superior incompleto & & $2,27 \%(\mathrm{n}=1)$ & \\
& Superior completo & $10,71 \%(\mathrm{n}=6)$ & $4,55 \%(\mathrm{n}=2)$ & \\
& Pós-graduação & $3,57 \%(\mathrm{n}=2)$ & & - \\
& & &
\end{tabular}

*Média e desvio-padrão

** Teste $t$ de Student

*** Teste Qui-Quadrado (X2) 
Tabela 2. Características clínicas dos pacientes oncológicos. Ijuí-RS, Brasil, 2015

\begin{tabular}{|c|c|c|c|c|}
\hline & & $\begin{array}{l}\text { Mulheres } \\
\qquad(\mathrm{n}=56)\end{array}$ & $\begin{array}{c}\text { Homens } \\
(\mathrm{n}=44)\end{array}$ & $\mathrm{p}$ \\
\hline \multirow{11}{*}{$\begin{array}{l}\text { Diagnóstico } \\
\text { primário }\end{array}$} & Boca & - & $6,82 \%(\mathrm{n}=3)$ & \multirow{11}{*}{$0,000 *$} \\
\hline & Garganta & $5,36 \%(\mathrm{n}=3)$ & $9,09 \%(\mathrm{n}=4)$ & \\
\hline & Intestino & $5,36 \%(\mathrm{n}=3)$ & $13,64 \%(\mathrm{n}=6)$ & \\
\hline & Leucemia & $3,57 \%(n=2)$ & $9,09 \%(n=4)$ & \\
\hline & Mama & $51,79 \%(\mathrm{n}=29)$ & - & \\
\hline & Ossos & $10,71 \%(n=6)$ & $4,55 \%(\mathrm{n}=2)$ & \\
\hline & Pele & - & $4,55 \%(\mathrm{n}=2)$ & \\
\hline & Próstata & - & $11,36 \%(\mathrm{n}=5)$ & \\
\hline & Pulmão & $7,14 \%(\mathrm{n}=4)$ & $15,91 \%(\mathrm{n}=7)$ & \\
\hline & Útero & $7,14 \%(\mathrm{n}=4)$ & - & \\
\hline & Demais órgãos***** & $8,92 \%(\mathrm{n}=5)$ & $25,00 \%(\mathrm{n}=11)$ & \\
\hline \multirow{2}{*}{ Metástase sistêmica } & Sim & $30,36 \%(\mathrm{n}=17)$ & $27,27 \%(\mathrm{n}=12)$ & \multirow{2}{*}{$0,735^{*}$} \\
\hline & Não & $69,64 \%(n=39)$ & $72,73 \%(\mathrm{n}=32)$ & \\
\hline \multirow{2}{*}{$\begin{array}{l}\text { Tratamento anterior } \\
\text { à quimioterapia }\end{array}$} & Cirurgia & $64,29 \%(\mathrm{n}=36)$ & $59,09 \%(n=26)$ & \\
\hline & Radioterapia & $42,86 \%(\mathrm{n}=24)$ & $43,18 \%(\mathrm{n}=19)$ & \\
\hline \multirow{9}{*}{$\begin{array}{l}\text { Sintomas } \\
\text { gastrointestinais }\end{array}$} & Constipação & $37,50 \%(n=21)$ & $25,00 \%(\mathrm{n}=11)$ & \\
\hline & Diarreia & $3,57 \%(\mathrm{n}=2)$ & $4,55 \%(\mathrm{n}=2)$ & \\
\hline & Disgeusia & $50,00 \%(\mathrm{n}=28)$ & $43,18 \%(\mathrm{n}=19)$ & \\
\hline & Inapetência & $57,14 \%(\mathrm{n}=32)$ & $36,36 \%(\mathrm{n}=16)$ & \\
\hline & $\begin{array}{l}\text { Mucosite/ } \\
\text { úlceras orais }\end{array}$ & $32,14 \%(\mathrm{n}=18)$ & $18,18 \%(\mathrm{n}=8)$ & \\
\hline & Náuseas & $48,21 \%(n=27)$ & $27,27 \%(\mathrm{n}=12)$ & \\
\hline & Vômitos & $41,07 \%(\mathrm{n}=23)$ & $22,73 \%(\mathrm{n}=10)$ & \\
\hline & Xerostomia & $67,86 \%(\mathrm{n}=38)$ & $54,55 \%(\mathrm{n}=24)$ & \\
\hline & Outros & $7,14 \%(\mathrm{n}=4)$ & $6,82 \%(n=3)$ & \\
\hline
\end{tabular}

* Teste Qui-Quadrado $\left(\chi^{2}\right)$

**** Demais órgãos citados por somente um dos pacientes oncológicos, como diagnóstico primário. 
Em relação aos principais casos de metástases sistêmicas, 8,93\% ( $\mathrm{n}=5)$ dos pacientes do sexo feminino apresentavam metástase nos ossos, 7,14\% (n=4) no fígado e 5,36\% (n=3) no pulmão; já entre o grupo do sexo masculino, 9,09\% ( $\mathrm{n}=4)$ apresentavam metástase no fígado, 4,55\% ( $\mathrm{n}=2)$ nos ossos, sendo destacado o mesmo índice para pulmão e pescoço.

Entre os participantes da pesquisa, mais de $75 \%$ necessitaram de algum tratamento anterior à quimioterapia - cirurgia e/ou radioterapia (tabela 2) -, sendo que somente $28,57 \%$ das mulheres e $18,18 \%$ dos homens não referiram terapia adicional. Ressalta-se que, do total de pacientes avaliados, apenas $8,93 \%(n=5)$ das mulheres e 6,82\% (n=3) dos homens relataram não apresentar nenhum sintoma ou efeito adverso do tratamento naquele período. Os sintomas gastrointestinais relatados estão demonstrados na tabela 2.

Na tabela 3, estão apresentados os aspectos referentes ao comportamento alimentar. A maioria dos indivíduos (80,36\% das mulheres e $84,09 \%$ dos homens) realizavam as refeições com familiares. Quanto a aversões alimentares desenvolvidas após o início do tratamento quimioterápico (32,14\% das mulheres e 27,27\% dos homens), houve predomínio de carnes, alimentos gordurosos, doces e alimentos sólidos. Em relação ao número de refeições após o início do tratamento, 37,5\% das mulheres e $31,82 \%$ dos homens aumentaram o fracionamento dietético para quatro ou mais refeições ao dia.

A tabela 4 apresenta os índices de consumo dietético da população estudada. Pacientes do sexo feminino consumiam em média 18,60 Kcal/Kg/dia e os do sexo masculino, 17,95 Kcal/Kg/dia. Houve diferença significativa entre mulheres e homens para o percentual de lipídeos $(p=0,044)$ e de carboidratos $(\mathrm{p}=0,026)$. 


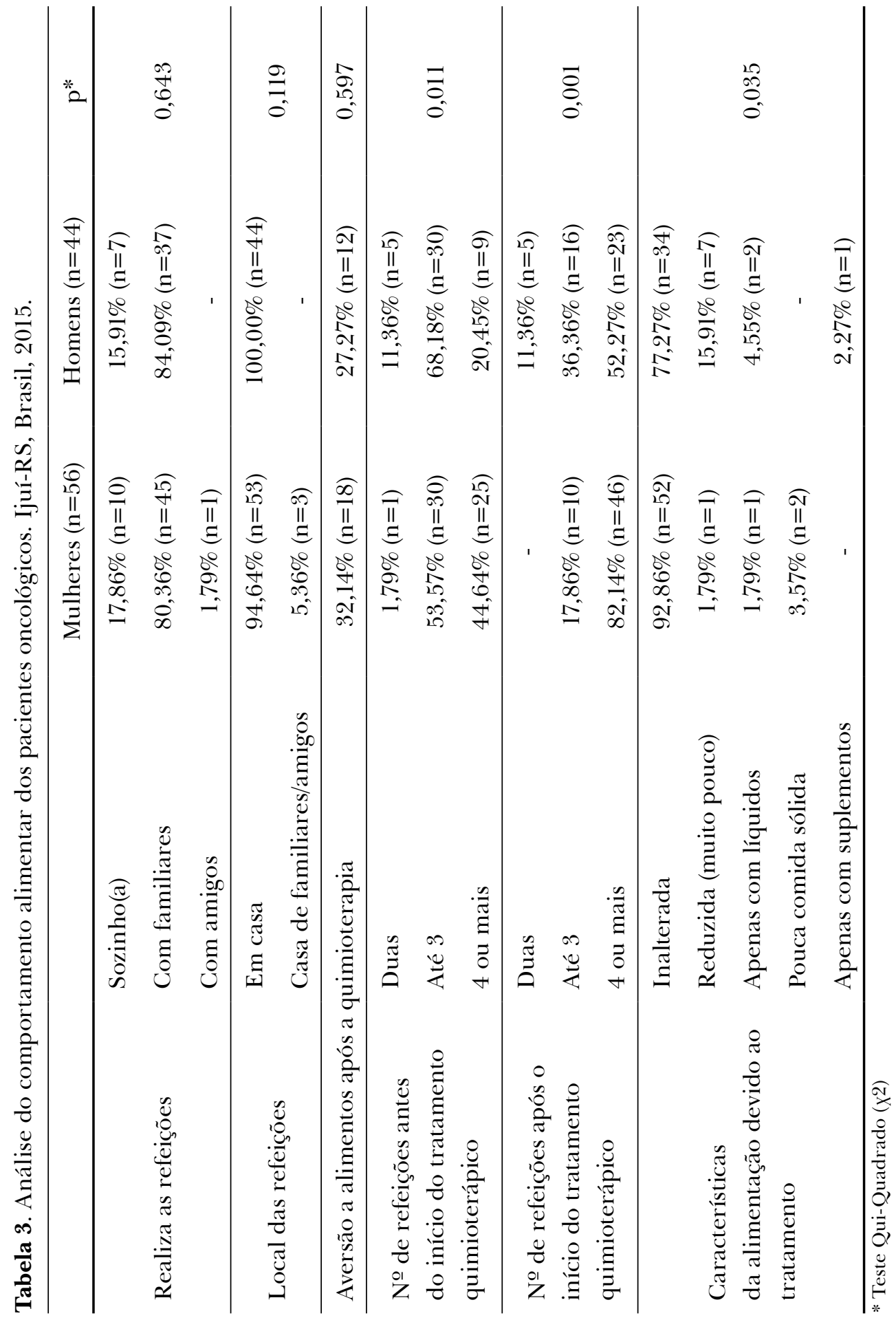


Tabela 4. Ingestão média de energia e macronutrientes dos pacientes oncológicos. Ijuí-RS, Brasil, 2015.

\begin{tabular}{lccc}
\hline & Mulheres $(\mathrm{n}=56)$ & Homens $(\mathrm{n}=44)$ & $\mathrm{p}^{*}$ \\
\hline Energia (Kcal) & $1213,99 \pm 278,62$ & $1292,86 \pm 327,69$ & 0,196 \\
\hline $\begin{array}{l}\text { Energia/Kg de peso } \\
\text { corporal (Kcal/kg) }\end{array}$ & $18,60 \pm 6,06$ & $17,95 \pm 4,53$ & 0,554 \\
\hline Proteína (g) & $59,03 \pm 16,84$ & $65,48 \pm 21,12$ & 0,092 \\
\hline Proteína (\%) & $19,64 \pm 4,90$ & $20,63 \pm 5,70$ & 0,355 \\
\hline $\begin{array}{l}\text { Gramas de proteína/kg } \\
\text { de peso corporal }(\mathrm{g} / \mathrm{Kg})\end{array}$ & $0,89 \pm 0,29$ & $0,91 \pm 0,31$ & 0,727 \\
\hline Lipídeos (g) & $36,60 \pm 13,76$ & $42,40 \pm 16,46$ & 0,057 \\
\hline Lipídeos $(\%)$ & $26,71 \pm 7,06$ & $29,92 \pm 8,72$ & 0,044 \\
\hline Carboidrato $(\mathrm{g})$ & $162,13 \pm 43,64$ & $162,34 \pm 55,41$ & 0,982 \\
\hline Carboidrato $(\%)$ & $53,65 \pm 8,12$ & $49,46 \pm 10,45$ & 0,026 \\
\hline Fibras (g) & $14,77 \pm 4,94$ & $13,29 \pm 7,17$ & 0,224 \\
\hline
\end{tabular}

* Teste $t$ de Student

Na tabela 5, estão descritos os dados antropométricos e suas respectivas classificações. O índice de massa corporal médio para o sexo feminino foi de $26,29 \mathrm{Kg} / \mathrm{m}^{2}$, e de $24,74 \mathrm{Kg} / \mathrm{m}^{2}$ para o sexo masculino. A circunferência da cintura foi de 79,44 cm e de $83,02 \mathrm{~cm}$, para mulheres e homens, respectivamente. Verificou-se que $50 \%(\mathrm{n}=28)$ das mulheres e $68,18 \%(\mathrm{n}=30)$ dos homens avaliados diminuíram o peso corporal nos últimos seis meses, enquanto o índice de elevação de peso foi de $32,14 \%(n=18)$ entre as mulheres e $18,18 \%(n=8)$ entre os homens. 
Tabela 5. Análise da avaliação antropométrica dos pacientes oncológicos. Ijuí-RS, Brasil, 2015

\begin{tabular}{|c|c|c|c|c|}
\hline & & $\begin{array}{l}\text { Mulheres } \\
(\mathrm{n}=56)\end{array}$ & $\begin{array}{c}\text { Homens } \\
(\mathrm{n}=44)\end{array}$ & $\mathrm{p}$ \\
\hline \multicolumn{2}{|l|}{ Peso usual $(\mathrm{kg})^{*}$} & $69,55 \pm 14,45$ & $76,80 \pm 15,20$ & $0,016 * *$ \\
\hline \multicolumn{2}{|c|}{$\begin{array}{l}\text { Mudança de peso nos } \\
\text { últimos } 6 \text { meses }(\mathrm{Kg})^{*}\end{array}$} & $6,13 \pm 4,71$ & $6,20 \pm 4,71$ & $0,933 * *$ \\
\hline \multicolumn{2}{|l|}{ Peso atual $(\mathrm{kg})^{*}$} & $68,11 \pm 14,80$ & $73,45 \pm 15,54$ & $0,082 * *$ \\
\hline \multicolumn{2}{|l|}{ Estatura $(m)^{*}$} & $1,61 \pm 0,08$ & $1,72 \pm 0,07$ & $0,000 * *$ \\
\hline \multicolumn{2}{|l|}{$\operatorname{IMC}\left(\mathrm{kg} / \mathrm{m}^{2}\right)^{*}$} & $26,29 \pm 5,01$ & $24,74 \pm 4,34$ & $0,107 * *$ \\
\hline \multirow{6}{*}{$\begin{array}{l}\text { Classificação } \\
\text { do IMC }\end{array}$} & Baixo-peso & $8,93 \%(\mathrm{n}=5)$ & $11,36 \%(\mathrm{n}=5)$ & \multirow{6}{*}{$0,458 * * *$} \\
\hline & Eutrofia & $30,36 \%(\mathrm{n}=17)$ & $45,45 \%(\mathrm{n}=20)$ & \\
\hline & Sobrepeso & $44,64 \%(\mathrm{n}=25)$ & $29,55 \%(\mathrm{n}=13)$ & \\
\hline & Obesidade grau I & $8,93 \%(\mathrm{n}=5)$ & $11,36 \%(\mathrm{n}=5)$ & \\
\hline & Obesidade grau II & $5,36 \%(\mathrm{n}=3)$ & $2,27 \%(\mathrm{n}=1)$ & \\
\hline & Obesidade grau III & $1,79 \%(\mathrm{n}=1)$ & - & \\
\hline Circunferência c & cintura $(\mathrm{cm})^{*}$ & $79,44 \pm 13,26$ & $83,02 \pm 16,20$ & $0,227 * *$ \\
\hline \multirow{3}{*}{$\begin{array}{l}\text { Classificação } \\
\text { circunferência } \\
\text { da cintura }\end{array}$} & Sem risco & $62,50 \%(n=35)$ & $79,55 \%(\mathrm{n}=35)$ & \multirow{3}{*}{$0,123 * * *$} \\
\hline & Risco aumentado & $19,64 \%(\mathrm{n}=11)$ & $6,82 \%(\mathrm{n}=3)$ & \\
\hline & $\begin{array}{l}\text { Risco muito } \\
\text { aumentado }\end{array}$ & $17,86 \%(\mathrm{n}=10)$ & $13,64 \%(n=6)$ & \\
\hline
\end{tabular}

*Média e desvio-padrão

** Teste $t$ de Student

*** Teste Qui-Quadrado $\left(\chi^{2}\right)$

\section{Discussão}

A quimioterapia compreende a administração de substâncias citotóxicas, sobretudo por via sistêmica, podendo apresentar diversas finalidades. ${ }^{16}$ No presente estudo, foi encontrada maior proporção de pacientes do sexo feminino, com baixo nível de escolaridade e casados, características semelhantes ao perfil sociodemográfico dos pacientes estudados por Palmieri et al. ${ }^{17}$ e Santos et al. ${ }^{18}$ Na população estudada, observou-se maior índice de diagnósticos primários de câncer de mama 
e pulmão, o que corresponde aos achados de Palmieri et al., ${ }^{17}$ segundo os quais as neoplasias de mama $(56,5 \%)$ e de pulmão $(17,4 \%)$ foram as mais frequentes.

O percentual de metástase na população deste estudo foi superior aos índices encontrados na avaliação do perfil clínico de idosos em tratamento antineoplásico, onde $49 \%$ dos casos apresentavam metástase, ${ }^{18}$ e na análise de pacientes de uma clínica especializada em relação à aceitação de preparações e sintomas decorrentes do tratamento oncológico, onde o índice de metástase foi de $17,4 \% .^{17}$

Vale ressaltar que os quimioterápicos em uso clínico usualmente ocasionam efeitos colaterais em virtude da toxicidade sobre as células, como náuseas, vômitos, lesões no esôfago, má nutrição, desequilíbrio hidroeletrolítico, entre outras consequências, que levam à redução da qualidade de vida e até mesmo à recusa dos pacientes em continuarem os ciclos quimioterápicos. ${ }^{16}$ Isso explica o motivo de apenas menos de $10 \%$ dos homens e mulheres avaliados não apresentarem sintomas decorrentes do tratamento antineoplásico.

Estudo de coorte realizado em Goiânia-GO por Silva et al. ${ }^{6}$ com 50 pacientes oncológicos adultos, de ambos os sexos, que estavam em tratamento quimioterápico, demonstrou que 38\% deles apresentavam pelo menos um sintoma associado ao tratamento, dados que corroboram os encontrados no presente estudo.

Prevaleceram as queixas de xerostomia, inapetência e digeusia dos alimentos por ambos os sexos, semelhante à análise de Ferreira, Guimarães e Marcadenti, ${ }^{3}$ que indicou número significativo de sintomas gastrintestinais, sendo os principais deles a inapetência (21\%) e a xerostomia (20\%). Em portadores de linfoma, a xerostomia apareceu como um dos sintomas de maior incidência após o início do tratamento quimioterápico, ${ }^{19}$ e entre pacientes de um hospital de Joinville-SC foi a segunda complicação de maior prevalência. ${ }^{20} \mathrm{Em}$ contrapartida, as náuseas foram os efeitos colaterais de maior frequência em ensaio clínico do tipo antes e depois, que avaliou 25 mulheres em São Paulo com câncer de mama e submetidas à quimioterapia, alcançando mais de $80 \%$ das pacientes. $^{21}$

O Inquérito Brasileiro de Nutrição Oncológica (IBNO), ${ }^{10}$ do ano de 2013, desenvolveu uma triagem em pacientes adultos com neoplasias malignas, internados em 45 instituições públicas e privadas do país, e verificou que os principais sintomas presentes foram inapetência, náuseas, xerostomia, disgeusia, constipação, diarreia e êmeses, sendo que apenas 14,2\% dos adultos e 12,5\% dos idosos não apresentavam sintomas, dados muito próximos aos achados do presente estudo.

Conforme se constatou na análise do comportamento alimentar, pacientes de ambos os sexos descreveram alterações correlacionadas às escolhas alimentares. Em relação às aversões alimentares adquiridas, mais de 50\% dos indivíduos indicaram aversão alimentar a um ou mais alimentos, dados que fortalecem os indícios de que, após o tratamento quimioterápico, há um impacto no 
comportamento alimentar. ${ }^{21}$ Estudos confirmam resultados semelhantes: análise da pesquisa desenvolvida por Silva et al. ${ }^{6}$ indicou que $38 \%$ dos pacientes avaliados também desenvolveram aversão alimentar a pelo menos um alimento, sendo que a carne vermelha apresentou o maior índice de rejeição, seguida por café, leguminosas, temperos e especiarias. Outro estudo constatou que subsequente ao tratamento, $52 \%$ dos pacientes indicaram no mínimo um alimento ou grupo ao qual desenvolveram rejeição alimentar, sendo que os alimentos gordurosos apresentaram o maior índice de aversão (38\%) e foram associados à sensação de incômodo e mal-estar; já as carnes foram citadas por apenas $7 \%$ dos indivíduos. ${ }^{21}$

Quanto ao número de refeições consumidas antes e após o início da quimioterapia, evidenciouse que a maioria dos indivíduos, de ambos os sexos, aumentou a frequência de ingestão para quatro ou mais refeições diárias após o início do tratamento. Esta mudança é considerada positiva clinicamente, pois de acordo com Pereira et al., ${ }^{22}$ o maior fracionamento dietético auxilia no controle do peso corporal, dos lipídeos séricos e da glicose sanguínea. Estudo realizado por esses autores para avaliar o fracionamento da dieta em relação ao perfil nutricional e de saúde de mulheres saudáveis demonstrou que o maior número de refeições diárias está associado a uma melhor qualidade alimentar. ${ }^{22}$ Consequentemente, supõe-se que alguns sintomas gastrointestinais decorrentes da terapêutica, que não foram evidenciados com predominância no estudo, podem estar sendo minimizados devido ao aumento do fracionamento da dieta dos pacientes.

No estudo, a caracterização da alimentação foi estatisticamente significante $(p=0,035)$, sendo que praticamente todas as mulheres e a maior parte dos homens definiram sua alimentação como inalterada, de forma similar ao Inquérito Brasileiro de Nutrição Oncológica, ${ }^{10}$ que constatou que $48,89 \%$ dos adultos avaliados não apresentavam problemas para se alimentar. A literatura aponta que, dependendo de como o paciente reage à patologia e ao tratamento, as alterações no comportamento alimentar podem ser evidenciadas e caracterizadas pela redução da ingesta alimentar ou pelo excesso, ${ }^{23}$ sendo esta uma possível hipótese para o fato de alguns pacientes terem relatado que, com o início do tratamento, passaram a se alimentar muito pouco ou apenas com líquidos, ou ainda com pouca comida sólida e apenas com suplementos nutricionais.

Um dado importante a ser discutido é o alto índice de pacientes que realizavam as refeições com familiares e em sua própria casa, o que pode ser um auxílio positivo ao tratamento, pois é comprovado cientificamente que a qualidade de vida e o comportamento alimentar estão correlacionados. ${ }^{21}$

Segundo Pinho et al., ${ }^{24}$ no paciente oncológico, as necessidades nutricionais são variáveis, pois sofrem interferência do grau de estresse, do tipo de tumor e da localização, da existência de má-absorção, entre outros aspectos. O Consenso Nacional de Nutrição Oncológica recomenda a ingestão energética de acordo com o estado nutricional do paciente, variando de 20 a $35 \mathrm{Kcal} /$ $\mathrm{Kg} /$ dia para pacientes em quimioterapia. ${ }^{9}$ Considerando estes parâmetros, evidencia-se que $\mathrm{o}$ 
consumo energético dos pacientes estudados está inadequado, pois os valores médios encontrados foram inferiores à recomendação mínima. Em outro estudo brasileiro, também foi observado que a maioria dos pacientes não atingiu as necessidades energéticas diárias. ${ }^{21}$

A ingestão média de energia total dos pacientes avaliados foi de aproximadamente $1.250 \mathrm{Kcal} /$ dia, dado inferior ao do estudo desenvolvido em Porto Alegre-RS com adultos em tratamento quimioterápico, que demonstrou consumo energético médio de $1.875 \mathrm{Kcal} / \mathrm{dia},{ }^{1}$ e da análise de Kolling e Santos ${ }^{25}$ em pacientes ambulatoriais com neoplasia de mama, que destacou ingestão média de $1.506 \mathrm{Kcal} / \mathrm{dia}$.

Tais resultados indicam que a contribuição percentual diária de lipídeos foi estatisticamente significante entre os sexos e inferior aos índices constatados por Kolling e Santos, ${ }^{25}$ em que o nutriente representou 34,5\% da dieta. Esses mesmos autores identificaram percentuais proteicos $(16,2 \%)$ superiores aos do presente estudo, e semelhante contribuição calórica de carboidratos $(49,3 \%) .{ }^{25}$ Ao comparar os dados com os índices da Pesquisa de Orçamentos Familiares (POF), ${ }^{26}$ que avaliou o perfil de consumo da população brasileira considerando o sexo e a faixa etária, os percentuais de lipídeos foram próximos aos observados pela POF para adultos e idosos (27\%); os índices proteicos foram superiores aos dados da POF, onde a ingestão variou entre $16 \%$ a 17\%; já a contribuição calórica média de carboidratos foi inferior à da POF, que variou de 54,8\% a 57,6\%.

As médias do consumo em gramas dos macronutrientes obtidas foram semelhantes aos índices apresentados por Santos et al. ${ }^{18} \mathrm{em}$ pesquisa com indivíduos idosos em quimioterapia, cujo consumo médio de carboidratos foi de 160,9 gramas/dia, 50,4 gramas/dia para proteínas e 33,1 gramas/dia para lipídeos. A ingestão média diária de fibras foi inferior aos achados da $\mathrm{POF},{ }^{26}$ que variaram de 17,6 a 23,5 gramas/dia, mas outras análises realizadas especificamente com pacientes oncológicos demonstraram resultado similar ao do presente estudo (em média 14 gramas/dia).,25 Estudo realizado com 182 mulheres com câncer de mama em tratamento antineoplásico ou não, no Ceará, indicou que a população avaliada consumia dieta hiperproteica, hipoglicídica e baixa em fibras, ${ }^{23}$ sendo o baixo índice de ingestão de fibras o único aspecto concomitante com a pesquisa.

Em relação ao consumo de gramas de proteína por quilograma de peso corporal, as médias encontradas foram menores que $1,0 \mathrm{~g} / \mathrm{kg} /$ dia para ambos os sexos. O Consenso Nacional de Nutrição Oncológica recomenda que, para pacientes em quimioterapia, a ingestão de proteínas deve variar de 1-2 g/kg/dia, dependendo do estresse e complicações clínicas. ${ }^{9}$ Sendo assim, pode-se afirmar que o consumo dos pacientes avaliados encontra-se abaixo do recomendado.

Salienta-se que Ambrosi et al. ${ }^{4}$ investigaram as alterações no consumo dietético de mulheres do sul do Brasil com câncer de mama e demonstraram significativo aumento na ingestão energética e de alguns macro e micronutrientes. Segundo Palmieri et al., ${ }^{17}$ é imprescindível a realização de mais estudos relacionados ao perfil dos pacientes oncológicos em atendimento ambulatorial, para compreender amplamente suas necessidades e aprimorar as terapias de aconselhamento nutricional. 
Portanto, as diferenças de consumo destacadas entre os estudos com indivíduos saudáveis ${ }^{26} \mathrm{e}$ este, realizado com indivíduos debilitados clinicamente, podem ser explicadas pelas prováveis alterações no padrão de ingestão devido ao tratamento.

Tratando-se da avaliação antropométrica, verificou-se um alto índice de alteração de peso em relação ao peso usual, sendo que a maioria dos indivíduos diminuíram o peso corporal nos últimos seis meses, à semelhança dos resultados do estudo de Dias et al., ${ }^{7}$ que evidenciaram prevalência da redução de peso em $55 \%$ dos participantes, associada à diminuição do consumo alimentar, fatores que elevam o risco de desnutrição.

Os dados observados ainda corroboram os achados de outros estudos, em que uma minoria de pacientes manteve o peso usual, sendo que tanto o aumento como a redução do peso apresentaram percentuais elevados, ${ }^{1,23}$ aspectos que podem ser consequência dos efeitos colaterais do tratamento. ${ }^{23}$ Da mesma maneira, estudo desenvolvido apenas com pacientes com neoplasia de próstata evidenciou que 95,5\% destes apresentaram mudança de peso, destacando índices de perda de peso levemente superiores aos de aumento. ${ }^{27}$

As consequências do tratamento oncológico estão vinculadas à disfunção gastrointestinal, que promove a diminuição da ingestão dietética e, consequentemente, perda de peso, ${ }^{9}$ sendo uma possível explicação para o alto índice de redução de peso destacado. Ressalta-se que o sintoma que precede o diagnóstico de desnutrição é a diminuição de peso não intencional. ${ }^{24}$

$\mathrm{Na}$ análise do IMC, destaca-se o sobrepeso entre as mulheres, seguido pelos índices de eutrofia; já entre os homens, o resultado foi o inverso. Os menores percentuais foram encontrados para obesidade graus II e III, em ambos os sexos. Em outras pesquisas, identificou-se o estado de eutrofia na maioria dos indivíduos avaliados. ${ }^{1,6}$ No entanto, resultados de Laffitte, Farias e Wszolek, ${ }^{19}$ Palmieri et al. ${ }^{17}$ e Sampaio et al. ${ }^{23}$ demonstraram o predomínio do excesso de peso na população estudada.

Oliveira et al. ${ }^{28}$ constataram maior índice de sobrepeso e obesidade associado com a alimentação inadequada, o que salienta a importância do acompanhamento nutricional. Segundo a literatura, a avaliação do IMC em pacientes com câncer de útero, mama e cólon demonstra maiores prevalências de sobrepeso; ' assim, a predominância do câncer de mama no presente estudo pode estar relacionada com o índice de sobrepeso superior entre as mulheres avaliadas.

Vale ressaltar, entretanto, que a desnutrição no paciente em tratamento quimioterápico pode ser ocultada por algumas medicações, como a terapia hormonal e os glicocorticoides, geralmente empregados de forma adicional à quimioterapia. ${ }^{1}$ Sendo assim, o índice de baixo-peso encontrado no estudo foi relativamente reduzido, similar aos dados destacados por outra pesquisa, em que apenas $6,3 \%$ dos indivíduos foram identificados como desnutridos. ${ }^{3}$ 
Sabe-se que a desnutrição em indivíduos com câncer é comum, e inúmeros fatores, além dos efeitos colaterais da própria terapia, determinam sua progressão, como o jejum prolongado para exames pré ou pós-operatórios, alterações no metabolismo (provocadas pelo tumor), maior necessidade nutricional para desenvolvimento da célula maligna e dificuldades mecânicas para mastigar e deglutir alimentos. ${ }^{9}$ Segundo Pinho et al. ${ }^{24}$ a prevalência do estado de desnutrição pode variar entre $30 \%$ e $80 \%$, conforme as características da célula tumoral.

Em relação à circunferência da cintura, a maioria dos pacientes, de ambos os sexos, foram classificados na categoria "sem risco associado às doenças cardiovasculares", diferentemente dos resultados dos demais estudos apresentados nesse segmento. Análise realizada por Oliveira et al. ${ }^{28}$ revelou que a maioria do público avaliado apresentava circunferência da cintura maior que $80 \mathrm{~cm}$, e estudo desenvolvido por Machado, Sampaio e $\mathrm{Lima}^{27}$ demonstrou médias maiores de circunferência da cintura entre homens com câncer, sendo que a maioria apresentava risco aumentado ou alto risco.

A prevalência da circunferência da cintura aumentada foi mais elevada entre as mulheres, assim como no estudo de Siqueira et al., ${ }^{29}$ no qual a obesidade abdominal entre o sexo feminino foi mais que o dobro da observada entre os homens. Felden \& Figueiredo ${ }^{30}$ observaram que, em pacientes com câncer de mama, 70\% apresentavam risco elevado para possível surgimento de patologias cardiovasculares, concluindo ainda que o acúmulo excessivo de gordura na cintura (> $88 \mathrm{~cm}$ ) é um preditor do câncer de mama.

Considera-se como limitação deste estudo o fato de a avaliação ter sido realizada em um único momento. O recordatório alimentar foi aplicado em apenas um dia, referente a um dia típico de semana, o que pode influenciar os resultados do consumo alimentar.

\section{Conclusões}

Os dados deste estudo identificaram que o tratamento quimioterápico altera o comportamento alimentar da maioria dos pacientes, devido principalmente aos efeitos colaterais da quimioterapia. Entretanto, pacientes do sexo feminino apresentaram menores índices de modificações na alimentação e maior fracionamento dietético antes e após o tratamento, o que fundamenta a hipótese de melhor qualidade alimentar entre as mulheres.

Observou-se que o sexo influencia no diagnóstico primário e no percentual energético de consumo diário de lipídios e carboidratos.

Em relação ao perfil nutricional, mesmo com a prevalência de pacientes em eutrofia e sobrepeso, o baixo consumo energético e proteico e o elevado índice de redução de peso corporal nos últimos seis meses sugerem possível situação de risco nutricional. 
Diante do exposto, percebe-se a importância do acompanhamento nutricional ao paciente oncológico em nível ambulatorial, incluindo como ação rotineira a avaliação nutricional e do comportamento alimentar, para definir possíveis condutas terapêuticas necessárias.

\section{Colaboradores}

Capelari P participou de todas as etapas, desde a concepção do estudo até a revisão da versão final do artigo; Ceni GC participou do desenho do estudo, da redação do artigo e da sua versão final.

\section{Referências}

1. Tartari RF, Busnello FM, Nunes CHA. Perfil nutricional de pacientes em tratamento quimioterápico em um ambulatório especializado em quimioterapia. Rev Bras Cancerol. 2010; 56(1):43-50.

2. World Health Organization. Relatório mundial sobre o câncer. Nota descritiva, n 297, fev. 2015. [Internet]. [acesso em: 02 dez. 2015]. Disponível em: http://www.who.int/mediacentre/factsheets/ fs $297 / \mathrm{es} /$

3. Ferreira D, Guimarães TG, Marcadenti A. Aceitação de dietas hospitalares e estado nutricional entre pacientes com câncer. Einstein. 2013; 11(1):41-46.

4. Ambrosi C, Di Pietro PF, Rockenbach G, Vieira FGK, Galvan D, Crippa CG, et al. Fatores que influenciam o consumo energético de mulheres no tratamento do câncer de mama. Rev Bras Ginecol Obstet. 2011; 33(8):207-213.

5. Comeau TB, Epstein JB, Migas C. Taste and smell dysfunction in patients receiving chemotherapy: a review of current knowledge. Support Care Cancer. 2001; 9(8):575-580.

6. Silva AM, Franco LP, Santos TSS, Passos XS, Costa BMF. Impacto das aversões alimentares no estado nutricional de pacientes oncológicos submetidos à quimioterapia. J Health Sci Inst. 2012; 30(2):166-170.

7. Dias VM, Barreto APM, Coelho SC, Ferreira FMB, Vieira GBS, Cláudio MM, et al. O grau de interferência dos sintomas gastrointestinais no estado nutricional do paciente com câncer em tratamento quimioterápico. Rev Bras Nutr Clín. 2006; 21(2):211-218.

8. Silva MPN. Síndrome da anorexia-caquexia em portadores de câncer. Rev Bras Cancerol. 2006; 52(1):59-77.

9. Brasil. Instituto Nacional de Câncer José Alencar Gomes da Silva. Consenso nacional de nutrição oncológica. 2 ed. rev. ampl. atual. Rio de Janeiro: INCA; 2015.

10. Brasil. Instituto Nacional de Câncer José Alencar Gomes da Silva. Inquérito Brasileiro de Nutrição Oncológica. Rio de Janeiro: INCA; 2013.

11. World Health Organization. Physical status: the use of and interpretation of anthropometry. Geneva: WHO; 1995. Technical report series, 854. 
12. Nacif M, Viebig RF. Avaliação antropométrica no ciclo da vida: uma visão prática. 2. ed. São Paulo: Metha; 2011.

13. Duarte ACG. Avaliação nutricional: aspectos clínicos e laboratoriais. São Paulo: Atheneu; 2007.

14. World Health Organization. Obesity: preventing and managing the global epidemic: report of a WHO consultation on obesity. Geneva: WHO; 2000. WHO Technical report series, 894.

15. Fisberg RM, Slater B, Marchioni DML, Martini LA. Inquéritos alimentares: métodos e bases científicas. Barueri: Manole; 2005.

16. Sawada NO, Nicolussi AC, Okino L, Cardozo FMC, Zago MMF. Avaliação da qualidade de vida de pacientes com câncer submetidos à quimioterapia. Rev Esc Enferm USP. 2009; 43(3):581-587.

17. Palmieri BN, Moulatlet EM, Buschinelli LKO, Silva MEMP. Aceitação de preparações e sua associação com os sintomas decorrentes do tratamento de câncer em pacientes de uma clínica especializada. Cad Saúde Coletiva. 2013; 21(1):2-9.

18. Santos CA, Ribeiro AQ, Rosa COB, Ribeiro RCL. Influência do gênero e do tipo de tratamento nos parâmetros nutricionais de idosos em oncologia. Rev Bras Cancerol. 2014; 60(2):143-150.

19. Laffitte AM, Farias CLA, Wszolek J. Sintomas que afetam a ingestão alimentar de pacientes com linfoma em quimioterapia ambulatorial. O Mundo da Saúde. 2015; 39(3):354-361.

20. Magnabosco Neto AE, Westphalen FH. Analysis of oral complications related to cancer therapy. Arch Oral Res. 2013; 9(2):159-164.

21. Verde SMML, São Pedro BMO, Mourão Netto M, Damasceno NRT. Aversão alimentar adquirida e qualidade de vida em mulheres com neoplasia mamária. Rev Nutr. 2009; 22(6):795-807.

22. Pereira LM, Vieira ALS, Santos PMHLC. Fracionamento da dieta e o perfil nutricional e de saúde de mulheres. Rev Nutr. 2014; 27(1):15-23.

23. Sampaio HAC, Oliveira NM, Sabry MOD, Carioca AAF, Pinheiro LGP. Influência do tipo de terapia antineoplásica sobre marcadores antropométricos e dietéticos em mulheres portadoras de câncer de mama. Rev Bras Cancerol. 2012; 58(2):223-230.

24. Pinho NB, Oliveira GPC, Correia MITD, Oliveira AGL, Souza CM, Cukier C, et al. Projeto Diretrizes. Terapia nutricional na oncologia [Internet]. Associação Médica Brasileira e Conselho Federal de Medicina; 2011. Disponível em: https://diretrizes.amb.org.br/_BibliotecaAntiga/terapia_ nutricional_na_oncologia.pdf

25. Kolling FL, Santos JS. A influência dos fatores de risco nutricionais no desenvolvimento de câncer de mama em pacientes ambulatoriais do interior do Rio Grande do Sul. Scientia Medica. 2009; 19(3):115-121.

26. Brasil. Instituto Brasileiro de Geografia e Estatística. Pesquisa de orçamentos familiares 2008-2009: análise do consumo alimentar pessoal no Brasil. Rio de Janeiro: IBGE; 2011. Disponível em: http:// biblioteca.ibge.gov.br/visualizacao/livros/liv50063.pdf

27. Machado SP, Sampaio HAC, Lima JWO. Caracterização antropométrica de portadores de câncer de próstata do Ceará, Brasil. Rev Nutr. 2009; 22(3):367-376. 
28. Oliveira DR, Carvalho ESC, Campos LC, Leal JA, Sampaio EV, Cassali GD. Avaliação nutricional de pacientes com câncer de mama atendidas no Serviço de Mastologia do Hospital das Clínicas, Belo Horizonte (MG), Brasil. Ciênc Saúde Coletiva. 2014; 19(5):1573-1580.

29. Siqueira DGB, Souza RKT, Mesas AE, Santos HG, Bortoletto MSS. Diferenças entre sexos nos determinantes da obesidade abdominal em adultos de 40 anos ou mais: estudo de base populacional. Rev Nutr. 2015; 28(5):485-496.

30. Felden JBB, Figueiredo ACL. Distribuição da gordura corporal e câncer de mama: um estudo de caso-controle no Sul do Brasil. Ciênc Saúde Coletiva. 2011; 16(5):2425-2433.

Recebido: 06 de setembro, 2017

Revisado: 16 de janeiro, 2018

Aceito: 09 de março, 2018 